\title{
Correlations Between Field Quality and Geometry of Components in the Collared Coils of the LHC Main Dipoles
}

\author{
B. Bellesia, V. Remondino, W. Scandale, E. Todesco, and C. Vollinger
}

\begin{abstract}
The Large Hadron Collider (LHC) [1], a proton-proton superconducting accelerator, will consist of about 8400 superconducting magnet units, all operating in superfluid helium at a temperature of $1.9 \mathrm{~K}$. The design of the superconducting main dipole magnets for the $\mathrm{LHC}$ is guided by the requirement of an extremely high field quality in the magnet aperture which is mainly defined by the layout of the superconducting coil and the position of the conductors. In order to avoid conductor movements within the magnet cross-section, the superconducting coils are held in place by surrounding stainless steel collars. In this paper, we review the dependence of field harmonics in the LHC main dipoles on dimensions of the hardware components of the collared coils.

An analysis of the dimensional measurements of these components which are used in the collared coils produced so far is given. Sensitivity tables which are worked out through a coupled magneto-static model give the variation of the multipoles on collars, copper wedge dimensions and cable geometry. A Monte Carlo method is used to simulate the effects of possible errors on the multipoles.
\end{abstract}

Index Terms-Field quality, magnetic measurements, multipoles, superconducting magnets.

\section{INTRODUCTION}

$\mathbf{T}$ HE magnetic field components in the aperture of a magnet are expressed in so-called multipoles which are the Fourier coefficients of the series expansion of the radial field component at a certain reference radius. The multipoles are given in units of $10^{-4}$ relative to the main field component. Since an extremely high field quality is needed in an accelerator magnet in order to store an intense particle beam for many hours, the relative deviation from the ideal field in the magnet's aperture should not exceed a few parts in units of $10^{-4}$. In addition, some field harmonics have to be controlled at a level of 0.1 units. The field errors in superconducting magnets are determined by the position of the conductors in the magnet cross-section, thus the manufacturing tolerances on all components have to be controlled within tight limits in order to keep the nominal position of the conductors in the magnet cross-section well determined.

Manuscript received October 20, 2003.

B. Bellesia, V. Remondino, W. Scandale and E. Todesco are with CERN, European Organization for Nuclear Research.

C. Vollinger is with the CERN, European Organization for Nuclear Research. She is also with the CERN, Accelerator Technology Department, Geneva, Switzerland (e-mail: Christine.Vollinger@cern.ch).

Digital Object Identifier 10.1109/TASC.2004.829052

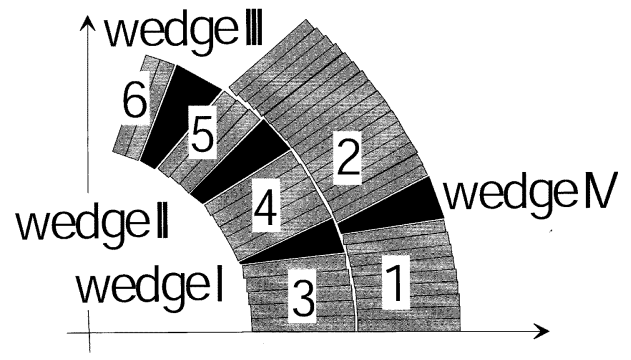

Fig. 1. First quadrant of the coil cross-section of one aperture of the LHC main dipoles.

\section{Method AND COLlaRed COIL COMPONENTS}

\section{A. Collared Coil Geometry}

The superconducting coils of the main dipoles consist of 6 blocks in two layers that are wound from Rutherford type cables of different thickness (cable 01 for the inner layer and cable 02 for the outer layer [1]). Both cables are slightly keystoned in order to give a good approximation of the ideal (cosine theta) distribution. Between the coil blocks, copper wedges are inserted to reach the desired field quality. Fig. 1 shows one quarter of a single coil cross-section with the four copper wedges (Roman numerals) and the cable blocks (Arabic numerals).

The coils for the two beam apertures are assembled within one common stainless steel collar and for all main dipoles a magnetic measurement at room temperature is carried out at this stage of assembly to detect field errors at an early stage of production. The outer contour of the coil cross-section is determined by the shape of the surrounding collars, hence errors in this shape will transform into a deformation of the outer coil shape. On the other hand, the azimuthal block positioning inside the coil cross-section is mainly dominated by the precise fabrication of the copper wedges. Since the main source of field errors are conductor displacements, we concentrate on mechanical tolerances of the collar geometry and of components inside the coil, i.e. the cable and the copper wedges.

\section{B. Monte Carlo Method}

We calculate the influence of geometrical tolerances of the collared coil components on the magnetic multipoles and derive sensitivity tables for all imperfections considered. From this, a 


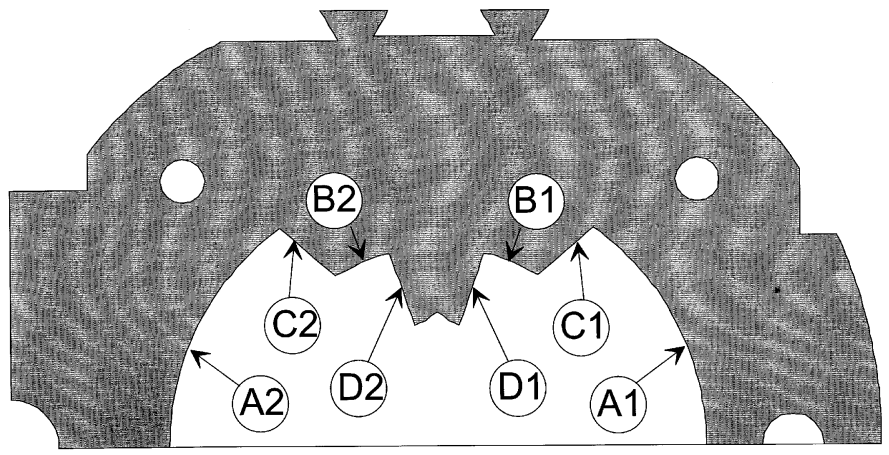

Fig. 2. Sketch of one quadrant of the collars including the space for one half coil geometry. The numbers indicate the region where the manufacturing tolerances are considered.

Monte Carlo method is used to simulate the effects of all possible errors arising from production tolerances of the components on the random and systematic part of the multipoles. For each imperfection, we simulate a Gaussian distribution with an average equal to the nominal value and the standard deviation equal to $1 / 6$ of the tolerance band. The calculated samples are $10^{6}$ for all components. In this way, we obtain the statistical properties of the induced magnetic multipoles.

\section{RESULTS}

\section{A. Collars}

1) Numerical Calculation: A numerical magneto-static model in the field computation program ROXIE [2] is used to determine the sensitivities of the harmonics on tolerances of the collars. In this way, a sensitivity matrix has been derived and is used in combination with the Monte Carlo simulation to estimate the impact on random errors of the field quality.

In this model, we assumed both the collars and the copper wedges to be infinitely rigid. In this way, the superconducting cable and the cable insulation absorb the changes in the collar's shape. In [3], it has already been shown that the main source of magnetic field errors is due to the internal part of the collars, whereas imperfections on the outer collar shape have little influence on the field quality in the aperture; thus they have been omitted. The coil cross-section of the dipoles is designed with a defined sextupole and decapole component in order to partly compensate coil magnetization effects at low field level. In addition, small systematic low even harmonics as b2 and b4 are produced at the stage of the collared coils resulting from the twin-aperture design [4]. Any effects of conductor displacements are expected to be visible in the measurements for the lower order multipoles.

In the numerical calculation, each part of the inner collar contributes in an independent manner and a violation of the left-right and up-down symmetry is to be expected. Therefore we avoided imposing symmetrical patterns to the imperfections of the inner contour of the collars with respect to the beam center. Fig. 2 shows one quarter of a collar piece indicating the areas where the sensitivities of the tolerances have been determined; the calculated values are shown in Table I for mechanical tolerances of $0.1 \mathrm{~mm}$ (in bold: sources of highest sensitivities).
TABLE I

Sensitivities of the Multipoles in Units at $17 \mathrm{~mm}$ ReFERENCE Radius, INDUCED BY MECHANICAL TOLERANCES OF $0.1 \mathrm{~mm}$

\begin{tabular}{lllllllr}
\hline \hline & $\begin{array}{l}\text { A1 } \\
\text { shift }\end{array}$ & \multicolumn{1}{l}{$\begin{array}{l}\text { A1 } \\
\text { tilt }\end{array}$} & $\begin{array}{l}\text { B1 } \\
\text { shift }\end{array}$ & \multicolumn{1}{l}{$\begin{array}{l}\text { C1 } \\
\text { shift }\end{array}$} & $\begin{array}{l}\text { C1 } \\
\text { tilt }\end{array}$ & \multicolumn{1}{l}{$\begin{array}{l}\text { D1 } \\
\text { shift }\end{array}$} & \multicolumn{1}{c}{$\begin{array}{l}\text { D1 } \\
\text { tilt }\end{array}$} \\
\hline$\Delta \mathrm{b} 2$ & $\mathbf{2 . 7 1}$ & $\mathbf{1 . 2 9}$ & -0.10 & 0.95 & -0.22 & 1.62 & -0.34 \\
$\Delta \mathrm{b} 3$ & 0.31 & $\mathbf{0 . 8 5}$ & -0.43 & 0.41 & -0.10 & 0.55 & -0.10 \\
$\Delta \mathrm{b} 4$ & -0.31 & 0.33 & $\mathbf{- 0 . 3 5}$ & 0.07 & -0.01 & -0.10 & 0.07 \\
$\Delta \mathrm{b} 5$ & $\mathbf{- 0 . 1 7}$ & 0.01 & 0.06 & -0.02 & 0.01 & -0.10 & 0.04 \\
$\Delta \mathrm{b} 6$ & 0.01 & -0.03 & $\mathbf{0 . 1 8}$ & -0.02 & 0.01 & 0.02 & -0.02 \\
$\Delta \mathrm{b} 7$ & 0.05 & 0.01 & 0.05 & -0.01 & 0.00 & 0.04 & -0.02 \\
\hline$\Delta \mathrm{a} 2$ & $\mathbf{- 3 . 9 2}$ & -1.34 & -0.52 & 1.04 & -0.16 & 1.22 & -0.13 \\
$\Delta \mathrm{a} 3$ & $\mathbf{- 2 . 4 2}$ & -1.00 & -0.34 & 0.08 & 0.01 & -0.13 & 0.11 \\
$\Delta \mathrm{a} 4$ & $\mathbf{- 0 . 9 3}$ & -0.75 & 0.22 & -0.10 & 0.04 & -0.16 & 0.06 \\
$\Delta \mathrm{a} 5$ & -0.22 & $\mathbf{- 0 . 4 0}$ & 0.28 & -0.05 & 0.01 & 0.06 & -0.03 \\
$\Delta \mathrm{a} 6$ & -0.05 & $\mathbf{- 0 . 1 3}$ & 0.02 & -0.01 & 0.00 & 0.07 & -0.03 \\
$\Delta \mathrm{a} 7$ & -0.06 & -0.04 & $\mathbf{- 0 . 1 0}$ & 0.00 & 0.00 & 0.00 & 0.01 \\
\hline \hline
\end{tabular}

TABLE II

Variation of the Multipoles Resulting From the Monte CARLo SiMULATION ON COLLARS AND FROM WARM MAGNETIC MEASUREMENTS TAKEN ON THE COLLARED COILS

\begin{tabular}{lcccccc}
\hline \hline & $\sigma \mathrm{b} 2$ & $\sigma \mathrm{b} 3$ & $\sigma \mathrm{b} 4$ & $\sigma \mathrm{b} 5$ & $\sigma \mathrm{b} 6$ & $\sigma \mathrm{b} 7$ \\
\hline calculated & 0.71 & 0.24 & 0.09 & 0.05 & 0.01 & 0.02 \\
measured & 0.86 & 1.70 & 0.12 & 0.62 & 0.04 & 0.26 \\
\hline & $\sigma \mathrm{a} 2$ & $\sigma \mathrm{a} 3$ & $\sigma \mathrm{a} 4$ & $\sigma \mathrm{a} 5$ & $\sigma \mathrm{a} 6$ & $\sigma \mathrm{a} 7$ \\
\hline calculated & 0.88 & 0.52 & 0.24 & 0.09 & 0.04 & 0.02 \\
measured & 1.28 & 0.43 & 0.35 & 0.10 & 0.08 & 0.06 \\
\hline \hline Expressed in nits at 17 mm ref radius
\end{tabular}

2) Monte Carlo Simulation: Starting from the sensitivity values in Table I, the allowed production tolerances of the collar pieces are:

- $\pm 15 \mu \mathrm{m}$ for the areas A1, B1 (both shifts and tilts)

- $\pm 25 \mu \mathrm{m}$ for the areas C1, D1 (both shifts and tilts).

The distribution is truncated at $3 \sigma$ to ensure that the tolerances are never exceeded. We applied shifts and tilts, assuming that straight lines are preserved and only the end-points can be displaced in the same direction (shift) or in opposite directions (tilt).

In areas $\mathrm{C} 1$ and $\mathrm{D} 1$, the shifts and the tilts affect the position of the mid-plane between the upper and the lower half of the coil in the respective layer. This effect is included in the simulation in such a way that the cable in the whole layer is affected by the shifts and tilts, respectively. Equally, shifts and tilts of the outer layer influence also the inner layer. These results are given in Table II.

Comparison between the measured and the calculated standard deviations (Table II) show that for all the cases investigated, the measured $\sigma$ is larger than the calculated one. The difference is biggest for the multipoles b3 and b5 whereas for the multipoles $\mathrm{b} 2$ and $\mathrm{b} 4$ the standard deviations of the measured and calculated multipoles are within the same range. Therefore, the precision of the production of the collars cannot account for all deviations in the multipoles observed in the measurements.

\section{B. Copper Wedges}

1) Production: Each copper wedge profile is delivered as 'batch' by the manufacturing firm. 


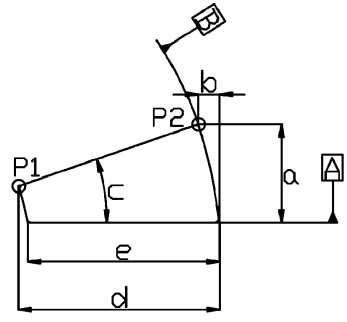

(a)

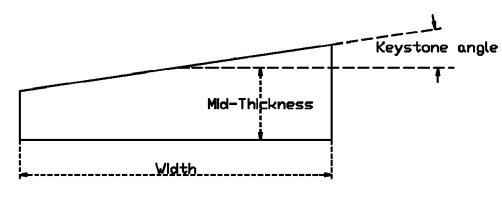

(b)
Fig. 3. Cu wedge (a) and cable (b) generic profiles and measured dimensions.

TABLE III

SENSITIVITY OF THE MULTIPOLES IN UNITS AT $17 \mathrm{~mm}$ REFERENCE RADIUS INDUCED BY COPPER WEDGE GEOMETRICAL TOLERANCES

\begin{tabular}{cccccc}
\hline \hline & $\Delta \mathrm{P} 1[\mathrm{~mm}]$ & $\Delta \mathrm{P} 2[\mathrm{~mm}]$ & $\Delta \mathrm{b} 3$ & $\Delta \mathrm{b} 5$ & $\Delta \mathrm{b} 7$ \\
\hline \multirow{2}{*}{ Prof I } & 0.000 & 0.030 & -0.2 & -0.002 & $\mathbf{0 . 0 1 4}$ \\
& 0.030 & 0.000 & -0.3 & -0.04 & $\mathbf{0 . 0 5 5}$ \\
\multirow{2}{*}{ Prof II } & 0.000 & 0.030 & 0.2 & $\mathbf{0 . 0 8}$ & -0.008 \\
& 0.030 & 0.000 & 0.4 & $\mathbf{0 . 1 5}$ & -0.036 \\
\hline \multirow{2}{*}{ Prof III } & 0.000 & 0.030 & $\mathbf{0 . 3}$ & -0.02 & -0.005 \\
& 0.030 & 0.000 & $\mathbf{0 . 6}$ & -0.08 & -0.008 \\
\hline \multirow{2}{*}{ Prof IV } & 0.000 & 0.030 & -0.1 & -0.001 & 0.002 \\
& 0.030 & 0.000 & -0.1 & 0.002 & 0.004 \\
\hline \hline
\end{tabular}

One batch contains 321 pieces which is sufficient for the assembling of ten dipoles plus one piece for destructive tests [5]. Three pieces per batch are measured: the first produced, the last produced, and an intermediate one. The dimensions that are measured are shown in Fig. 3(a).

The position of the surface between P1 and P2 in Fig. 3(a) is the crucial parameter of our analysis; it has to be located inside a tolerance band of $\pm 30 \mu \mathrm{m}$, in the assumption that the sides of the wedges are straight lines. The control protocols of the copper wedge production show batches within the tolerances with some isolated exceptions especially for profile III (see Fig. 1). It is found that the two first batches manufactured (which are the batch used for the prototype magnets, PROT, and the batch A) are geometrically different from the successive production (see [6] for details).

2) Numerical Calculation: We evaluate the sensitivity of each wedge dimension on magnetic field multipoles in the hypothesis of an infinite elastic modulus. We assume that cable blocks absorb the overall effect of copper wedge imperfections, keeping the same external shape of the coil (see Table III).

We considered the separated effects of each copper wedge, giving to the pair $[\Delta \mathrm{P} 1, \Delta \mathrm{P} 2]$ of each profile the maximal allowed configurations: $[0,0.030] \mathrm{mm}$ and $[0.030,0] \mathrm{mm}$ and we computed the induced magnetic effects. Profile III has the strongest influence on b3, while b5 is mostly affected by Profile II. The largest effect on b7 is given by Profile I. Profile IV has only an influence on b3.

By using traceability [6], we computed in each dipole the expected effect of wedge imperfections and subtracted it from the measured harmonics. This way, we reduced the measured multipoles to the nominal edge dimensions (Table IV). The main effect is a large reduction of the trend of b3 which had been observed in the first dipoles. All these dipoles are done from batch A (see [6] for details).
TABLE IV

AVERAge and Standard DeViation of MEAsured Multipoles in the Collared CoIls, RaW ( $b_{n}$ meas.) AND Post-PROCESSED VAlues $\left(\mathrm{b}_{\mathrm{n}}-\Delta \mathrm{b}_{\mathrm{n}}\right)$ WHERE THE EFFECT OF WEDGES ARE TAKEN OUT

\begin{tabular}{lcccc}
\hline \hline & \multicolumn{2}{c}{ Old coil cross-section } & \multicolumn{2}{c}{ New coil cross-section } \\
\hline & meann & $\sigma$ & meann & $\sigma$ \\
\hline $\mathrm{b}_{3}$ meas. & 1.1 & $\mathbf{1 . 9}$ & -1.9 & 1.1 \\
$\mathrm{~b}_{3}-\Delta \mathrm{b}_{3}$ & 1.0 & $\mathbf{1 . 6}$ & -2.2 & 1.2 \\
\hline $\mathrm{b}_{5}$ meas. & 1.18 & 0.42 & 0.41 & 0.39 \\
$\mathrm{~b}_{5}-\Delta \mathrm{b}_{5}$ & 1.22 & 0.43 & 0.45 & 0.40 \\
\hline $\mathrm{b}_{7}$ meas. & 0.631 & 0.161 & 1.126 & 0.085 \\
$\mathrm{~b}_{7}-\Delta \mathrm{b}_{7}$ & 0.648 & 0.164 & 1.130 & 0.087 \\
\hline \hline
\end{tabular}

TABLE V

GeOMETRICAL Dimensions AND TOLERANCES OF CABLES

\begin{tabular}{cccc}
\hline \hline $\begin{array}{c}\text { Cable } \\
\text { type }\end{array}$ & $\begin{array}{c}\text { Mid thickness } \\
{[\mathrm{mm}]}\end{array}$ & $\begin{array}{c}\text { Keystone } \\
\text { angle }[\mathrm{deg}]\end{array}$ & Width [mm] \\
\hline 01 & $1.9 \pm 0.006$ & $1.25 \pm 0.05$ & $15.1[-0 ;+0.08]$ \\
\hline 02 & $1.48 \pm 0.006$ & $0.9 \pm 0.05$ & $15.1[-0 ;+0.08]$ \\
\hline \hline
\end{tabular}

This means that a relevant part of the initial trend in b3 was due to the out-of-tolerance of the copper wedges of batch $\mathrm{A}$. The spread in b3 is reduced from 1.9 to 1.6 units when the effect of copper wedge is taken out, whereas the situation of b5 and b7 is not affected.

3) Monte Carlo Simulation: The imperfections are distributed with a standard deviation of $1 / 4$ of the total tolerance band of $60 \mu \mathrm{m}$ to simulate a bigger measurement spread (see for details [6]). From the simulation we get a spread for b3 of 0.41 units, b5 of 0.08 units and for b7 of 0.03 units. Comparison with the values in Table II show that wedge tolerances are not the main source of the random component measured in the manufactured dipoles: they account only for $1 / 3$ to $1 / 5$ of these measured values (Table II).

\section{Cables}

1) Production: The cables, delivered at CERN in four unit lengths per pallet (a unit length is the length needed to wind a layer) have to satisfy several control tests [5]. We will analyze the geometrical measurements of the two types of cables, all of trapezoidal shape. The three geometrical measured quantities are the mid-thickness, keystone angle and width (see Fig. 3(b) and Table $\mathrm{V}$ for the respective tolerances).

CERN performs dimensional tests on one cable unit length over four from each pallet delivered. The cable production is within tolerances and is very homogeneous among the manufacturer [7].

2) Numerical Calculation: We evaluate the influence of the cable tolerances on the field harmonics. The variation of the mid-thickness affects the odd normal multipoles by a change of the pre-stress [8] and the even skew multipoles by a shift of the mid plane [9].

A difference of the keystone angle between the upper layer cable and the lower one results in a tilt of the contact surface between the two poles. Changes in the keystone angle only affect the even skew harmonics. This study was carried out with a magneto-static model assuming an infinitely rigid collar cavity and copper wedges. From the sensitivity matrix given in [9], it 
TABLE VI

SENSITIVITY OF THE MULTIPOLES IN UNITS AT $17 \mathrm{~mm}$ REFERENCE RADIUS INDUCED BY CABLE GEOMETRICAL TOLERANCES

\begin{tabular}{|c|c|c|c|c|c|c|}
\hline & \multicolumn{2}{|c|}{ Mid Thickness } & \multirow{2}{*}{$\begin{array}{l}\text { K-stone } \\
\text { m.p. tilt }\end{array}$} & \multicolumn{2}{|c|}{ Width } \\
\hline & & pre stress & m.p. shift & & thinner & wider \\
\hline \multirow{2}{*}{ 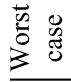 } & u.p. & $+0.006 \mathrm{~mm}$ & $+0.006 \mathrm{~mm}$ & $0.05 \mathrm{deg}$ & $-0.02 \mathrm{~mm}$ & $0.02 \mathrm{~mm}$ \\
\hline & 1.p. & $+0.006 \mathrm{~mm}$ & $-0.006 \mathrm{~mm}$ & $-0.05 \mathrm{deg}$ & $0 \quad \mathrm{~mm}$ & $0 \quad \mathrm{~mm}$ \\
\hline \multirow{5}{*}{$\begin{array}{l}\stackrel{\dot{\Phi}}{\grave{\Xi}} \\
\frac{\bar{\Phi}}{\Xi} \\
\vdots\end{array}$} & $b_{3}$ & -0.5 & 0 & 0 & 0.11 & -0.11 \\
\hline & $\mathrm{b}_{5}$ & 0.13 & 0 & 0 & -0.004 & 0.004 \\
\hline & $\mathrm{b}_{7}$ & -0.020 & 0 & 0 & 0.004 & -0.004 \\
\hline & $a_{2}$ & 0 & 4.8 & -2.0 & 0.72 & -0.73 \\
\hline & $\mathrm{a}_{4}$ & 0 & 0.72 & -0.23 & 0.10 & -0.10 \\
\hline \multirow{5}{*}{ 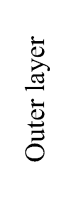 } & $\mathrm{b}_{3}$ & -0.8 & 0 & 0 & -0.12 & -0.19 \\
\hline & $b_{5}$ & 0.21 & 0 & 0 & 0.03 & 0.00 \\
\hline & $\mathrm{b}_{7}$ & -0.034 & 0 & 0 & -0.005 & -0.004 \\
\hline & $\mathrm{a}_{2}$ & 0 & 4.7 & -1.8 & 1.1 & -1.6 \\
\hline & $\mathrm{a}_{4}$ & 0 & 0.34 & -0.23 & 0.31 & -0.19 \\
\hline
\end{tabular}

can be seen that the deviations of the cable width give an effect on all considered harmonics.

All results are shown in Table VI evaluating the effects of the tolerances according to the worst case. The cable dimensions also have a relevant influence on the systematics, e.g., if all upper cables are larger than the lower ones, but within tolerances, one can obtain both $\mathrm{a} 2$ and $\mathrm{a} 4$ outside the allowed ranges for the systematics (2.0 units for a 2 and 0.26 for a4). On odd normal multipoles the impact is rather small when compared to the allowed range for the systematics given by the beam dynamics ( 7.0 for b3, 0.7 for b5 and 0.5 for b7). For b5, however a shift of up to 0.35 units can be obtained in case that the cables are systematically at the edge of the mid-thickness tolerance.

3) Monte Carlo Simulation: The standard deviation and the mean used here is resulting from the statistics of each manufacturer [7]; we refine the simulation by using a "typical" spread of the geometrical measurements along the cable length. In addition, we analyze the effect of two poles done by two different cable produces within one coil.

We found a negligible effect on random components with respect to both measurements and to targets [10] (one order of magnitude less), with the exception of the effects of cable thickness on a2 (up to 1.25 units [ $1 \sigma]$ against 1.9 specified for the collared coil). Assuming that inner and outer layer effects are independent, the simulation shows that the cable mid-thickness could account for most of the specified a2.

When two poles manufactured with cables from two different producers are coupled, the effect on the odd normal multipoles is negligible. The effect on the even skew multi-poles can be large. The imperfections of the cable mid-thickness produces an average effect on the random part of a 2 of about one unit, whereas those of the keystone angle result in 0.5 units. On the random part of a4 we get 0.07 units due the mid thickness and 0.06 units due to the keystone angle. The effects of the cable width are negligible. Hence, if the combinations of different manufacturers for the upper and lower poles were systematic during production, it would generate systematic even skew multipoles out of
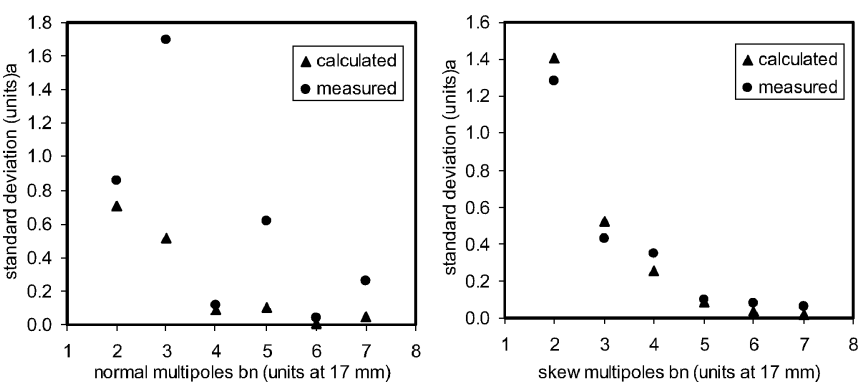

Fig. 4. Combination of the results of the Monte Carlo simulations of Section III; measured values as dots, calculated values as triangles.

specifications. However, the effect on a single or on a few magnets does not affect the systematic over the entire machine nor the random component.

\section{COMBINING THE RESUlTS}

Combining the results derived in Section III (see Fig. 4) it can be seen that all $\sigma$ for the normal even multipoles are still far below the measured $\sigma$ shown in Table II. This shows that the tolerances of the collared coil components do only partially account for multipole deviations that are observed during production. For the other low order multipoles, we can see a good agreement. Exceptions are the skew multipoles a 2 and a3, where the calculated $\sigma$ are slightly higher than the measured ones. It can be seen in the Tables I and VI, and in Fig. 4, that these two multipoles mainly arise from variations in the collar region $\mathrm{A} 1$ and from the cable thickness. We therefore assume that at least one of these tolerances within the production are kept with greater precision than demanded in the specifications. This would explain the slight overestimation of the error that is seen when the total tolerance band has been used for the calculations.

\section{ACKNOWLEDGMENT}

The authors wish to thank F. Bertinelli, M. Cornelis, P. Fessia, and A. Musso for providing data for the traceability analysis and R. Wolf and I. Vanenkov for the support given to this work.

\section{REFERENCES}

[1] The LHC study group, , Geneva, CERN/AC/95-5, 1995.

[2] S. Russenschuck et al., "Integrated design of superconducting accelerator magnets," Jour. Phys. AP 1, pp. 92-102, 1998.

[3] W. Scandale et al., "Influence of mechanical tolerances on field quality in the LHC main dipoles," IEEE Trans. Appl. Supercond., vol. 10, no. 1, pp. 73-76, March 1999.

[4] P. Fessia et al., "Selection of the cross-section design for the LHC main dipole," IEEE Trans. Appl. Supercond., vol. 10, no. 1, pp. 65-68, March 1999.

[5] "LHC Technical Specification," CERN, Int. Report, LHC-MB-C1-0006.

[6] B. Bellesia et al., "Influence of Copper Wedge Dimensions on Field Harmonics in the Main LHC Dipoles,", LHC Project Report 630, 2003.

[7] B. Bellesia, "Investigation on Cable Dimensions,", in progress.

[8] P. Ferracin, PhD Thesis, CERN THESIS 2002-009.

[9] S. Pauletta, Master Thesis, Torino University, 2002.

[10] S. Fartoukh and O. Bruning, "Field Quality Specification for the LHC Main Dipole Magnets," CERN, Geneva, Switzerland, LHC Pr. Report 501, 2001. 